Año 26

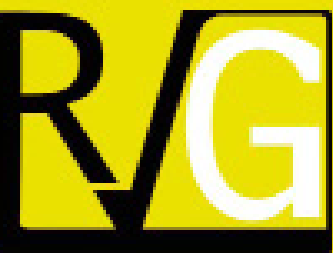

Número Especial 5, 2021

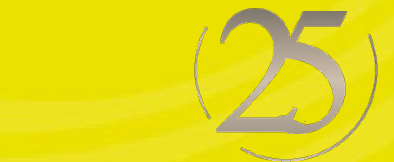

ANMTSARO
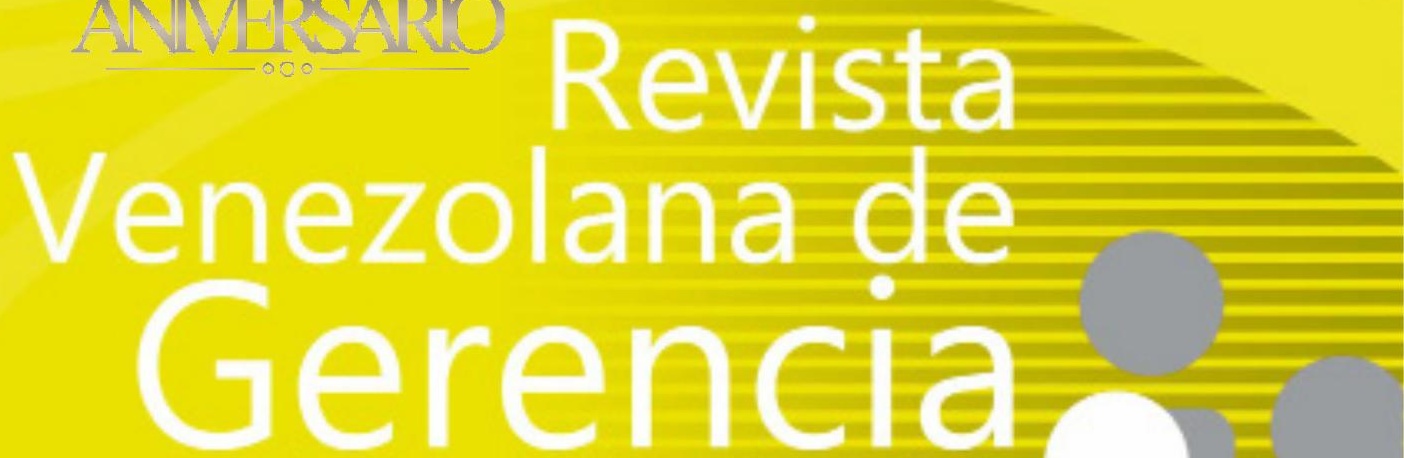
COMO CITAR: Galarza-Iglesias, A. M., Ordoñez-Hernández, C. A., y GomézSalazar, L. (2021). Labor reinstatement of leaders of transportation companies. Revista Venezolana De Gerencia, 26(Número Especial 5), 563-577. https://doi. org/10.52080/rvgluz.26.e5.36
Universidad del Zulia (LUZ)

Revista Venezolana de Gerencia (RVG)

Año 26 Número Especial 5 2021, 563-577

ISSN 1315-9984 / e-ISSN 2477-9423

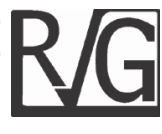

\title{
Reintegro laboral de líderes de empresas de transporte
}

\author{
Galarza-Iglesias, Ana M.* \\ Ordoñez-Hernández, Cecilia A.** \\ Goméz-Salazar, Lessby***
}

\section{Resumen}

El reintegro laboral es la condición en la cual un trabajador retorna al trabajo después de una ausencia por afectación en salud que restringe el desarrollo pleno de actividades laborales. Con el objetivo de comprender el significado del reintegro laboral para líderes y supervisores en una empresa de bus de rápido tránsito en Cali- Colombia, se llevó a cabo esta investigación de tipo cualitativa con enfoque desde fenomenología hermenéutica. Se contó con 8 participantes a quienes se les realizó entrevista semiestructurada en profundidad. Como resultados se tiene que para los líderes y supervisores el reintegro laboral tiene un significado de obligatoriedad que afecta el sistema productivo. Se percibe dependencia, desconocimiento y relación de desconfianza hacia el trabajador y al sistema de seguridad social. La comunicación en el reintegro es deficiente. Esta investigación permite concluir que los líderes y supervisores deben crear vínculos efectivos con los trabajadores reintegrados y el sistema de seguridad social a partir de un modelo teórico que contemple las necesidades particulares. Así mismo, el conocer la estructura y significado del reintegro laboral aporta fundamentos para generar sistemas integrados en las empresas y políticas públicas que garanticen trabajo digno de todo ser humano, prevención de la discapacidad articulado a un sistema productivo.

Palabras clave: Reintegro laboral; fenomenología; Bus de rápido tránsito; restricciones laborales.

Recibido: 20.02.21

Aceptado: 15.05.21

* Doctora en Salud de la Universidad del Valle. Terapeuta Ocupacional, Mag. administración educativa, esp. Pedagogía, - Universidad del Valle, Cali, Colombia. Facultad de Salud, Terapia Ocupacional. ORCID: https:// orcid.org/0000-0001-5643-3700. autor correspondencia: ana.galarza@correounivalle.edu.co

** Doctora en ciencias de la salud y el trabajo de la Universidad de Guadalajara. Fisioterapeuta, Mag. Salud Ocupacional, Universidad del Valle, Cali, Colombia. ORCID: https://orcid.org/0000-0001-9296-3768

*** Doctora en ciencias biomédicas de la Universidad del Valle. Fisioterapeuta, Mag. Salud Ocupacional. Grupo de investigación en Biomecánica, Universidad del Valle, Cali, Colombia. Email: lessby.gomez@ correounivalle.edu.co. ORCID: https://orcid.org/0000-0003-2338-9410 


\title{
Labor reinstatement of leaders of transportation companies
}

\begin{abstract}
Work reinstatement is the condition in which a worker returns to work after an absence due to a health condition that restricts the full development of work activities. In order to understand the meaning of job reinstatement for leaders and supervisors in a rapid transit bus company in Cali- Colombia, this qualitative research was carried out with a hermeneutical phenomenology approach. There were 8 participants who underwent an in-depth semi-structured interview. As a result, for leaders and supervisors, reinstatement has a mandatory meaning that affects the production system. Dependence, ignorance and a relationship of mistrust towards the worker and the social security system are perceived. Communication in the refund is poor. This research allows us to conclude that leaders and supervisors must create effective links with reintegrated workers and the social security system based on a theoretical model that considers particular needs. Likewise, knowing the structure and meaning of labor reintegration provides foundations to generate integrated systems in companies and public policies that guarantee decent work for every human being, prevention of disability articulated to a productive system.
\end{abstract}

Key words: Return to work, restrictions, Rapid Transit Bus, employer, work occupation, management.

\section{Introducción}

En toda organización el capital humano es considerado clave para el logro de metas y por lo tanto de su desarrollo productivo (Simancas, et al, 2018)independientemente de si son grandes empresas o pequeñas y medianas empresas. El propósito de este artículo es explorar, desde la perspectiva documental, la relación entre el capital humano y la productividad en las pequeñas y medianas empresas. Como insumo se revisaran las diferentes etapas de evolución en este proceso, así como los elementos que aumentan el potencial para la productividad empresarial. Así mismo se procede a revisar la realidad productiva de las Pymes industriales, comerciales y de servicios en Barranquilla; para ello se tomó como referencia el informe de resultados del primer semestre de 2016 de la Encuesta Gran Pyme Colombiana (ANIF, 2016a, 2016b. El concepto de capital humano abarca, no solo el conocimiento y habilidades de un trabajador, sino también la forma en que trabaje y su salud (Becker, 1965). Es así que cuando se presenta una condición de salud que genera restricciones médicas (Espinosa \& Morris, 2002) (Waddell et al, 2010) (Ministerio de la Protección Social, n.d.) o pérdida de capacidad laboral en un trabajador (Organización Mundial de la Salud, 2014) se afecta su participación en el entorno laboral y por lo tanto se requiere realizar un proceso de reintegro laboral (Asociación Nacional de Empresarios de Colombia 
[ANDI], 2017).

Alrededor de este tema existen políticas que obligan a los empleadores a ofrecer todas las garantías para que el trabajador sea reintegrado nuevamente a su trabajo (Lopéz \& Chicana, 2017) la presente contribución al debate sobre el futuro del trabajo pretende llamar la atención sobre la idoneidad de impulsar un nuevo planteamiento de la discapacidad en su relación con el trabajo que responda, de forma adecuada, a las características y requerimientos de tres colectivos vulnerables y necesitados de una especial tutela para su inserción en el ámbito laboral en igualdad de condiciones que los demás: las personas que sufren enfermedades raras, las que se ven afectadas por enfermedades mentales, y las mujeres con discapacidad. Conversación a la que se dirige: Trabajo decente para todas las personas Sumario: 1. Trabajo y discapacidad: nuevos retos 2. La inserción social y laboral de las personas con enfermedades raras 3 . La inserción social y laboral de las personas con enfermedades mentales 4 . La inserción social y laboral de las mujeres con discapacidad 1 Vicerrectora de Responsabilidad Social, Transparencia e Igualdad de la Universidad de Murcia; Investigadora Principal del Proyecto DER2016-76557-R I"El Futuro del sistema español de protección social. Análisis de las reformas en curso y propuestas para garantizar su eficiencia y equidad ( $V$ según la capacidad valorada por la calificación de pérdida de capacidad laboral y ocupacional (Ministerio de la Protección Social Dirección General de Riesgos Profesionales, 2010), (Colombia, 2014). Existen diversos modelos de reintegro que plantean guias para lograr reintegros efectivos (Trillos, Tolosa
\& Escobar, 2016) (Schultz \& Editors, 2016) (Simonelli, et al, 2010) siendo un fenomeno complejo a partir del hecho que en cada uno se tiene objetivos diferentes, que si bien en algunos momentos pueden complementarse en otras ocasiones pueden ser contrarios.

En general para la mayoría de las empresas lograr el reintegro de un trabajador se constituye en un reto $y$ es particularmente difícil en empresas cuya actividad económica se centra en una sola tarea como es el caso de los conductores de Bus Rápido Transito- BRT, en las cuales el $90 \%$ de los trabajadores contratados se desempeña como operador de bus limitando seriamente las posibilidades de la empresa de ofrecer alternativas de reintegro al trabajador en tareas diferentes.

De un buen proceso de reintegro laboral depende la permanencia del trabajador en su trabajo, si esto no se logra, se presenta el riesgo tener un proceso fallido (Ministerio de la Protección Social Dirección General de Riesgos Profesionales, 2010), (Zondek, 2015), que puede generar o aumentar el grado de discapacidad, disminuir la participación social y ocupacional (Cuervo Díaz, 2016) del trabajador y afectar todo un sistema productivo con implicaciones económicas y de compromiso social.

El papel del empleador se basa en la gestión para realizar el reintegro laboral dando un rol activo a partir de asignación de tareas y responsabilidades acorde con la capacidad laboral del trabajador, (Awang \& Mansor, 2018; Ask \& Magnussen, 2015)(2. Desde el conocimiento y comprensión del significado atribuido a la reincorporación se logra una aproximación a la realidad del fenómeno que puede aportar 
información para la construcción de nuevas políticas tanto al interior de la empresa como a nivel nacional e internacional enfocadas a procesos que fortalezcan las condiciones de trabajo de la población en general a la vez que se mantenga la productividad en la empresa.

Para lograr dicha aproximación existen diferentes metodologías, una de ellas la fenomenología la cual pretende dar respuesta a preguntas relacionadas con la estructura y esencia de las experiencias vividas de un fenómeno para una persona $\mathrm{o}$ un grupo de personas.

Estudio cualitativo de carácter fenomenológico hermenéutico que explora el significado de la experiencia cotidiana vivida para las personas (Creswell, 2013; Husserl, 2000; Leonard, 1989; Morse, 2005; Patton, 2002), considerando los cuatro existenciales básicos: espacio vivido, cuerpo vivido , tiempo vivido y relaciones vividas (León, 2009; Merlau-Ponty, 2000).

La empresa donde se realizó la investigación pertenece al sistema de transporte masivo integrado, el cual abastece a cerca del $93 \%$ de la ciudad de Santiago de Cali-Colombia. En su estructura organizacional cuenta con área de gestión gerencial, gestión de operaciones, gestión de flota, gestión humana, gestión administrativa y financiera y el sistema integrado de gestión donde $90 \%$ de los trabajadores tienen cargo de operador de bus. El transporte masivo mundialmente es referido como autobús de tránsito rápido -. ATR o, BRT por sus iniciales en inglés (Bus Rapid Transit).

El grupo de estudio estuvo conformado por el total de líderes y supervisores de la empresa mencionada, que representaron en total
8 participantes. Se realizó entrevista semiestructurada con guion según los referentes teóricos (Hernández, Fernandéz, \& Baptista, 2010; Patton, 2002; Zainal, 2007).

Las entrevistas se realizaron de manera personal ( $\mathrm{J}$ Taylor $\mathrm{R}$ Bogdan, 2000). Fueron grabadas y luego se escucharon y transcribieron textualmente. La sistematización y análisis de los datos obtenidos se realizó con el apoyo del software ATLAS. Ti (versión 8.0).

Se realizó análisis fenomenológico interpretativo en sus tres fases: En la primera se realizó lectura y relectura de las entrevistas y se describieron los hallazgos en términos de experiencia y comportamiento, opinión/valor, sentimientos, conocimiento y lo sensorial. En la segunda fase de tematización o reducción fenomenológica, se determinaron los elementos esenciales de la descripción, se codificaron teniendo en cuenta los cuatro existenciales fenomenológicos; en la tercera fase de interpretación, se especificó el sentido de la experiencia vivida por los líderes y supervisores integrando los hallazgos referentes al fenómeno de reintegro laboral.

\section{Reintegro laboral: algunas ideas teóricas}

El reintegro laboral es conocido de diferentes formas: Reincorporación laboral, reintegro laboral $\mathrm{o}$ retorno al trabajo. A nivel internacional está definido como el proceso por el cual una persona logra compensar con el mayor grado posible las desventajas originadas de una deficiencia o una discapacidad, que afecta su desempeño laboral, dificultándole o impidiéndole 
la integración socio laboral mediante la consecución, el mantenimiento y la promoción de una vida productiva (Ministerio de la Protección Social, 2019).

En el reintegro laboral participan actores diferentes, la empresa, el trabajador, el sistema de salud, los compañeros de trabajo, la familia, cada uno con objetivos diferentes, que pueden o no coincidir en sus intereses (Ask \& Magnussen, 2015; Awang \& Mansor, 2018; Schultz \& Editors, 2016; Simonelli, et al, 2010; Trillos Ch et al, 2016). Sobre el tema, algunos autores mencionan que la ausencia de salud y bienestar afectan el sentido de la autoeficacia en el trabajador y por tanto, afecta su proceso de retorno al trabajo (Etuknwa y Daniels $\mathrm{K}, 2019)$.

El papel del empleador es clave y se basa en la gestión que se realiza al dar directriz para realizar las adaptaciones, estrategias de apoyo que requiera el trabajador con la promoción de lugares de trabajo saludables con asignación de tareas y responsabilidades acorde con su capacidad laboral, procesos desde la interacción en el trabajo y de autogestión de las salud, buena comunicación y trabajo en equipo con el médico tratante (Ask \& Magnussen, 2015; Awang \& Mansor, 2018); otros factores que también se recomienda considerar para mantener un buen ambiente y evitar acoso laboral a causa del reintegro laboral, son los relacionados con las características propias del trabajador, la interacción con las personas en el contexto laboral y el ambiente laboral (Carvajal \& Davila, 2013).

A partir de la mirada de los supervisores, investigaciones han indagado sobre la disponibilidad y capacidad que tiene la empresa para contratar personas con discapacidad; además realizaron análisis de puestos de trabajo y concluyen que la empresa es el único facilitador en el proceso de inclusión laboral pues es la que dispone de los medios para realizarla; además se evidenció que solo $14 \%$ de las empresas cumple con la ley de cuotas en Brasil (Simonelli et al, 2010).

\section{Aproximaciones metodológicas del estudio}

Se contó con 8 participantes. con edad mínima 28 años y máxima 54 años. Respecto al género, 3 mujeres y 5 hombres. Todos con estudios superiores y 4 con posgrado. Los nombres fueron reemplazados por códigos protegiendo la identidad. Los resultados se presentan en la tabla 1. 
Tabla 1

\section{Características sociodemográficas generales de los participantes en la investigación}

\begin{tabular}{cccc}
\hline Participante & Edad (años) & Género & Escolaridad \\
\hline 01 Rosa & 36 años & Femenino & Pregrado \\
\hline 02 Pedro & 35 años & Masculino & Posgrado \\
\hline 03 Juan & 44 años & Masculino & Pregrado \\
\hline 04 David & 22 años & Masculino & Tecnológico \\
\hline 05 María & 23 años & Femenino & Pregrado \\
\hline 06 Pablo & 54 años & Masculino & Posgrado \\
\hline 07 Claudia & 28 años & Femenino & Posgrado \\
\hline 08 Felipe & 32 años & Masculino & Posgrado \\
\hline
\end{tabular}

Fuente: elaboración propia

\subsection{Fase de descripción fenomenológica}

En esta fase se utilizó el esquema propuesto por Patton (2002), considerarondolas siguientes categorías: Experiencia y comportamiento: describe las acciones, actividades y vivencias de los participantes referente al reintegro laboral; la opinión-valor, aborda las opiniones, deseos, intenciones, valores propios o de otros, evidencia lo que los participantes piensan sobre el reintegro, la categoría de sentimientos refleja el sentir de los participantes ante el reintegro; el conocimiento identifica el dominio de los participantes sobre el tema de estudio y abarca el conocimiento académico y el empírico; lo sensorial considera lo visto, oído, escuchado, percibido por los participantes en el estudio y que esté relacionado el reintegro laboral.

\section{- Experiencia y comportamiento}

Los líderes viven el reintegro laboral a partir de actividades que deben ejecutar para que el trabajador cumpla con funciones acorde a las directrices de la empresa respetando las restricciones médicas. Desde su experiencia el proceso es difícil y no puede ser realizado por una sola persona, requiere, además de recursos económicos y de trabajadores disponibles, del apoyo del del trabajador, otros profesionales, empleador,

de las entidades del sistema general de riesgos laborales y de salud. Así mismo consideran que el reintegro afecta la productividad en la empresa.

[...]Eso significa a nivel empresarial una perdida de productividad... si una persona no puede cumplir el $100 \%$ las funciones, lo que hago es que tengo que tener a otra persona que reemplace ese porcentaje que no esta haciendo o el total de la persona .. con la empresa contratamos operadores para que trabajen 8 horas al día que es la jornada laboral establecida y hay personas que tienen restricciones de que trabajan solo 4 horas y tengo, pues tengo que tener otra persona que me complete las 4 
horas y sería un costo adicional o tengo personas que no pueden desempeñarse como operadores sino que tengo que llevarlos a otra, reubicarlos en otra área que posiblemente yo no este necesitando ese cargo [...]Pedro

Sienten poco apoyo y participación de la entidad prestadora de salud (EPS) y la administradora de riesgos laborales o mutual (ARL), sin embargo, perciben que los casos que se encuentran tramitados a través de la ARL son más sencillos de llevar precisamente porque hay mayor posibilidad de comunicación y menor tiempo en la respuesta a las solicitudes del trabajador y de la empresa. La retroalimentación es solo a través de los trabajadores generando confusión en temas tanto del pronóstico de la condición de salud del trabajador como en lo referente a las restricciones laborales. Esto se da por desconocimiento del contexto laboral y las exigencias del cargo; lo que suele ser aprovechado por el trabajador para obtener ganancias secundarias tanto en tiempo de incapacidad como en recursos económicos y disminución de carga laboral. En general los participantes consideran que la comunicación entre las EPS y ARL con la empresa debe mejorar para tener mayores posibilidades de éxito en el proceso.

[... [ dependemos de la información que nos de la persona, nosotros como empresa no tenemos acceso a historias clínicas.. no es fácil comunicarse con los médicos de las EPS, no puedo, si una persona me dice "no, es que estoy pidiendo la cita y no me la dan", pues ..me quedo con esa información " [...] Pedro

El proceso de reintegro se asume como un requisito legal que debe cumplirse por lo que cuando no hay un puesto de trabajo definido donde se pueda hacer el reintegro, se le asigna al trabajador tareas que pueden carecer de valor no solo para él mismo sino también para la empresa.

[...] uno se encuentra que sí, la empresa cumple... los pone a hacer otra labor, pero está llena de conductores que no operan,....el cumplimiento de la norma legal eso si se hace acá porque digamos a las personas las ponen a hacer algo, o sea, no las mandan para la casa... entonces eso se cumple $y$ los trabajadores muchas veces están contentos con eso... pero aquí en la ansiedad, en el ambiente laboral es que él, muchas veces el mismo trabajador las siente porque se siente inútil [...]Pablo

\section{- Opiniones y valores}

El proceso de reintegro laboral requiere del esfuerzo del trabajador y la empresa. Valoran el rol del jefe inmediato o supervisor para realizar la gestión de adaptación de funciones en el nuevo cargo y reconocen que algunos no están en disposición de hacerlo. Sin embargo, dan mayor responsabilidad de los avances y éxito al trabajador, quien con su actitud se compromete o no al aceptar y realizar las funciones que le sean asignadas.

[...] hay otros que vienen con una restricción en la mente y en el alma $y$ en el corazón entonces, de ese tipo de personas me cuido.. porque uno trabaja con gente que viene aquí que viene con bastón, con muletas que con uno de estos señores que no han sabido valorar lo que es tener la vida y en mi equipo tuve de estas personas como todo comienzo, bonito, agradable, ya después se dice que pelan el cobre [...]Juan

Desde su perspectiva en el proceso de reintegro hay tres grupos de trabajadores: los que tienen una 
condición de salud y afectada su funcionalidad, los que además tienen una afectación emocional y los que simulan enfermedad para no retomar al trabajo, se escudan en las restricciones médicas para no asumir las tareas, magnifican sus limitaciones para obtener incapacidades y restricciones que les impidan retomar al cargo de operador, y tener jornadas laborales más cortas, lo que genera un mal ambiente de trabajo y la pérdida de confianza con el equipo de reintegro.

[...] he identificado, gente verdaderamente enferma que no progresa sin causa explicable, gente que se enferma después de que se golpea, se accidenta y no se les ve pues las ganas de seguir trabajando y los que definitivamente están aprovechados de la situación y que son víctimas, se victimizan, víctimas del sistema, víctimas de la empresa, de todo y no quieren trabajar [...] Felipe

\section{- Sentimientos}

Los líderes manifiestan que han sido testigos de que los compañeros de trabajo discriminan, ponen sobrenombres lanzan comentarios ofensivos que atentan contra la integridad del trabajador reintegrado. Estos comportamientos les genera inconformidad, pero no hacen nada para evitarlos se asume éste como un comportamiento normalizado entre hombres conductores.

[...]yo lo he visto con algunos, como que los degradan, lo denigran entonces ellos... obviamente no se van a sentir bien y el entorno laboral también es muy importante para el proceso de recuperación y de rehabilitación de un trabajador, entonces si lo otros compañeros lo están chuzando, lo están puyando, le estén diciendo cosas ofensivas y dicen "no, es que este no sirve pa' nada sino para archivar" y cosas así, entonces lo que hace en el trabajador es bajar la guardia y no le dan ganas de ir a rehabilitación [...]Juan

Los participantes sienten frustación y ansiedad, al ser responsables del proceso de reintegro y tener que aplicar restricciones y recomendaciones médicas alejadas del contexto, sienten presión de parte del trabajador que puede no estar satisfeccho con las funciones que se le reasignan y por la empresa que espera productividad del trabajador y finalmente temor de tomar desiciones que pueden afectar el estado de salud del trabajador.

[...]cuando una persona viene para reincorporación laboral en lo primero que pienso es: ¿Qué tendrá y dónde lo voy a poner? Ese es como el primer impacto que yo digo "Pucha, ¿dónde voy a poner esta persona para que sea productiva pero que no se afecte su recuperación o su estado de salud actual"[...]David

\section{- Conocimiento}

Los líderes conocen la normativa colombiana de obligatoriedad para el reintegro la cual es ajustada según los recursos al interior de las empresas a traves de un protocolo que permiten operativizarlo cumpliendo con la norma, la productividad y conservando la salud del trabajador. Deben conocer los puestos de trabajo, no por lo que dice el trabajador sino en el propio contexto laboral, unido a la condición de salud del trabajador y las restricciones médicas.

[...] es muy común, a veces el médico tratante clínico, ortopedista, fisiatra, neurocirujano, etc., emite juicios sin saber ni siquiera que hace el trabajador[...] entonces nosotros lo que hacemos es por ejemplo, le dicen "no 
vaya a levantar peso, ni vaya a hacer fuerza, ni vaya a empujar, ni vaya a brincar, ni vaya a subir nada" pero eso no aplica acá, entonces lo que nosotros hacemos acá es tener en cuenta algunas de esas restricciones que ellos dan de acuerdo a la función que tiene cada trabajador, por ejemplo aquí la persona ni salta, ni brinda, ni sube gradas, ni hace fuerza, ni levanta peso, eso no va ahi en las restricciones sino que van ahí las que sean pertinentes para las tareas que hace el trabajador[...]Pablo

\section{- Lo sensorial}

El reintegro laboral se convierte para los líderes en una carga dificil de llevar porque existe ante todo el amparo de la norma y tanto la empresa como para la ARL y la EPS se quedan sin herramientas para que el proceso sea integral y con los trabajadores que realmente lo necesitan.

[...]el sistema muy proteccionista como todo a nivel legal ...finalmente toda la carga la tenemos los empleadores y debe haber una forma de desenmascarar a esas personas para poder, que sea limpio el proceso de reintegro[...] Pedro

\subsection{Segunda Fase: reducción fenomenológica de las vivencias y experiencias del proceso de reintegro laboral}

Se categorizó y codificó los hallazgos. En el cuadro 1 se presenta el consolidado con la lista de categorias y códigos. Postermente, al terminar el cuadro se presenta la descripción de los códigos.

\begin{tabular}{|c|c|c|}
\hline $\begin{array}{l}\text { ategorías, código } \\
\text { mpleadores que p }\end{array}$ & $\begin{array}{l}\text { Cuadro } 1 \\
\text { le los cuatro exist } \\
\text { icipan en el proce }\end{array}$ & $\begin{array}{l}\text { ciales básicos } \\
\text { o de reintegro }\end{array}$ \\
\hline Componente Fenomenológico & Categoria & Códigos \\
\hline & & Apoyo en gestión \\
\hline & Con el empleador & Falta de apoyo \\
\hline & & Desinterés \\
\hline & & Desconfianza \\
\hline & & Manipulación \\
\hline & $\begin{array}{l}\text { Con trabajadores en proceso } \\
\text { de reintegro }\end{array}$ & Interés personal \\
\hline Rolacinnoc vividac & & Ganancia secundaria \\
\hline Relaciones vividas & & Exigencia \\
\hline & & Relación de Lejanía \\
\hline & Con entidadec FPS $\vee A R$ & Relación Limitada \\
\hline & Con entiaades Ers y ARL & Comunicación poco clara \\
\hline & & Obligatoria \\
\hline & Otron thohainda 10 & Relación Inexistente \\
\hline & Utros trabajadores & Relación Conflictiva \\
\hline
\end{tabular}


Galarza-Iglesias, Ana M.; Ordoñez- Hernández, Cecilia; Gómez-Salazar, Lessby

Reintegro laboral de líderes de empresas de transporte

\section{Cont... Cuadro 1}

\begin{tabular}{lll}
\hline \multirow{2}{*}{ Cuerpo vivido } & $\begin{array}{l}\text { Imaginario de la sensación } \\
\text { vivida en el cuerpo }\end{array}$ & Pesadez \\
\cline { 3 - 3 } Tiempo vivido & $\begin{array}{l}\text { Tiempo en función del proce- } \\
\text { so de reintegro laboral }\end{array}$ & $\begin{array}{ll}\text { Fentitud } \\
\text { Espacio vivido }\end{array}$ \\
\hline
\end{tabular}

Fuente propia: elaboración a partir de hallazgos.

\section{- Relaciones Humanas vividas}

La relación humanas se perciben desde el apoyo para seguir un protocolo y cumplir la norma, desde el desinteres de jefes intermedios para ejecutar el plan de acción y asignar funciones nuevas a los trabajadores reintegrados. La relación con los trabajadores reintegrados es desconfiada, se presume que los trabajadores manipulan y en algunos casos exigen ganancias secundarias amparándose en la base normativa de la obligación por parte del empleador de mantener el vinculo laboral. Las exigencias se dan en horarios, actividades, tipo de vehiculo a conducir y funciones que seran asignadas.

[...]no solamente es una restricción médica sino que esconden detrás de esa restricción médica, se amparan atrás de una mentira o esa condición para hacer peticiones fuera de contexto[...]Juan

Con las entidades como Entidad Prestadora de Salud y Administradora de Riesgos Laborales o mutual, las relaciones son percibidas lejanas, limitadas y poco participativas requiriendo de un intermediario para que se puedan dar, en especial, para facilitar la comunicación. Estas relaciones son importantes y obligatorias para un buen desarrollo del proceso y es necesario estrecharlas para que sean de ayuda

\section{- Cuerpo vivido}

El reintegro laboral se percibe por el lider como algo pesado, una carga que exige esfuerzo y los resultados dependen del trabajo en equipo, sin recompensa. Se sienten maniatados, obligados a cumplir con una normativa o los intereses de los trabajadores.

[...]el sistema muy proteccionista como todo a nivel legal finalmente toda la carga la tenemos los empleadores $y$ debe haber una forma de desenmascarar a esas personas para poder, que sea limpio el proceso de reintegro, que las personas estén ahí, tengo toda la conciencia de que es que no puede trabajar y es que haya una voluntad genuina[...]Maria

\section{- Tiempo vivido}

Se sienten afectados por el excesivo tiempo que se toman las entidades EPS y ARL para hacer la atención y seguimiento a los trabajadores lo que retrasa el proceso de reintegro sin posibilidades de mejorar la situación por 
el escaso nivel de comunicación entre estas y la empresa. El reintegro es una tarea adicional a las que desempeñan en sus cargos, requiere tiempo sobre todo para realizar el seguimiento en la evolución del trabajador.

[...]realmente acercamientos con las EPS para mirar cómo le va en la evolución, si podemos agilizar algo pero realmente no es fácil esto y requiere de recurso, recurso es de tiempo y de profesionales, de la voluntad también de las EPS y a veces nos encontramos muchas barreras de todo tipo, burocráticas en el caso de las EPS que se demoran mucho[...] Pedro

\section{- Espacio vivido}

La empresa se considera un lugar de aprendizaje, hay un espacio en el que los trabajadores que estan reintegrados sin una tarea fija se ubican voluntariamente, deben cumplir el horario de 8 horas, sin embargo las tareas que se les asignan pueden tomar máximo cuatro, por lo que los trabajadores vagan por la empresa o se sientan en este lugar a esperar a que pase el tiempo.

[...] aquí es una universidad de reintegro trabajar aquí porque aquí están todos los matices que puede ver uno en este tema [...]Pablo

\subsection{Tercera Fase del analisis fenomenológico: interpretación}

En esta fase se hizo una aproximación lo mas cercana posible a la realidad vivida de los participantes a partir de sus relatos.

El significado atribuido por los participantes al reintegro laboral está enmarcado como una acción obligatoria segun la normativa para mantener el vinculo laboral de un trabajador con alteracion en su salud y por lo tanto, con restricciones laborales que disminuyen su productividad y afectan la empresa. El reintegro laboral depende del sistema de salud y de riesgos laborales quienes emiten las restricciones laborales descontextualizadas y dificiles de aplicar, desconociendo la realidad de la empresa y las exigencias del puesto de trabajo y del trabajador con su condición de salud y actitudes asumidas frente al proceso que no siempre son positivas y pueden estar afectadas por las ganacias secundarias.

La falta de claridad y respuesta oportuna de la EPS y ARL sobre lo que sucede con el trabajador y la actitud del trabajador durante el proceso genera sentimientos de impotencia ante la responsabilidad que les ha dado $y$ una mirada de desconfianza frente al trabajador, el proceso de reintego es una carga que se les ha asignado sin autonomia.

Al ser el empresario un intermediario y sin embargo, los responsables del reintegro, finalmente son los trabajadores quienes a partir de sus actitudes, compromiso, evolución médica, podrán retomar sus actividades productivas relacionadas con su cargo original.

Finalmente,con los resultados de este estudio constrastados con estudios anteriores y teorias se tiene que el reitengro laboral exige un trabajo articulado en equipo a partir del conocimiento y experiencia de quienes participan del proceso (Ntsiea \& van Aswegen, 2017; Fernández et al, 2009). Es una necesidad que en la actualidad no se está cubriendo y genera conflicto desde el mismo momento que el trabajador presenta una condicion de salud que genera restricciones para 
trabajar.

Desde el sistema productivo los lideres y supervisores manifiestan preocupacion por la productividad evidenciando un conflicto entre el reintegro laboral y los indicadores economicos. El sentido de obligatoriedad (Colombia, 2002; Ministerio de la Protección Social, n.d.) con el cual es tomado el reintegro, ofrece las pautas generales para el reintegro laboral, sin embargo, desconoce la particularidad del contexto y genera sentimientos de soledad, impotencia, frustración manifestando incorfomidad al tener un vinculo laboral con un trabajador que está afectando la productividad y clima laboral.

Así mismo se evidencia una relación de desconfianza la cual afecta la relación entre lideres y supervisores con el trabajador lo cual genera otro aspecto a trabajar desde la empresa para generar cultura que permita la interacción y comunicación desde la confianza (Guinot \& Barghouti, 2019). En respuesta a esta situación la empresa ajusta la norma en un protocolo propio evidenciando la oportunidades de mejora para las politicas del reintegro laboral desde el mismo marco normativo (Cuervo-Díaz, Moreno-Angarita, \& Elizabeth, 2017), el de seguridad social con las ARL y EPS asi como al interior del sector empresarial y la condicion de salud del trabajador.

La actitud del trabajador es considerada como un factor clave dando la posibilidad de un buen proceso de reintegro laboral. Mas allá de la edad, del conocimiento y la condición de salud (Muijzer, et al, 2012), si el trabajador está dispuesto, el reintegro se gestiona de manera exitosa con la expectativa de que se retome el cargo original y aporte completamente en el sistema productivo.
El conocimiento de la condición de salud y como puede afectar la capacidad laboral del trabajador relacionada con el puesto de trabajo específico es básica para comprender hasta que punto los trabajadores pueden ser asignados a ciertas funciones y las adaptaciones que se deben realizar en el puesto de trabajo.

\section{Conclusiones}

Los líderes y supervisores son auto-concebidos como intermediarios entre el trabajador y la normativa, desconociendo su papel relevante.

Es necesario crear vínculos efectivos entre los actores del reintegro general a partir de un modelo teórico que contemple las situaciones particulares y necesidades del empleador, el trabajador y el sistema de salud De un buen proceso de reincorporación laboral depende la permanencia del trabajador en su trabajo, si esto no se logra se presenta el riesgo tener un proceso fallido que puede generar además de costos al sistema de riesgos laborales un impacto en el trabajador, su contexto familiar, laboral y social. También, la empresa se ve afectada no solo en su sistema productivo, sino en sus indicadores de sistemas de gestión integrado y responsabilidad social. El conocimiento de la estructura del fenomeno del reintegro laboral aporta fundamentos que permitan generar sistemas integrados de gestión en la empresa y políticas públicas encaminadas a garantizar trabajo digno de todo ser humano, considerado en los objetos de desarrollo del milenio.

Existen dos miradas según la intencionalidad de los profesionales y las entidades del sistema : una desde la salud como tal proceso saludenfermedad con el objetivo de mejorar 
la condición de salud desconociendo la actividad laboral del trabajador y otra, desde la relación de la capacidad laboral con el puesto de trabajo, siendo está última la mirada que permite abordar el reintegro laboral y donde el papel de los lideres, superviores es relevante.

\section{Referencias bibliográficas}

Asociación Nacional de Empresarios de Colombia ANDI. (2017). Salud y estabilidad en el empleo: retos jurídicos y económicos para la sostenibilidad de las empresas. Medellín. http://www.andi.com.co/ Uploads/ANDI\%20-\%20Salud $\% 20$ y\%20estabilidad $\% 20$ en $\% 20$ el $\% 20$ empleo.pdf

Ask, T., \& Magnussen, L. H. (2015). Supervisors' Strategies to Facilitate Work Functioning among Employees with Musculoskeletal Complaints: A Focus Group Study. The Scientific World Journal, 865628. https://doi. org/10.1155/2015/865628

Awang, H., \& Mansor, N. (2018). Predicting Employment Status of Injured Workers Following a Case Management Intervention. Safety and Health at Work, 9, 347-351. https://www.ncbi.nlm.nih.gov/pmcl articles/PMC6129999/pdf/main.pdf

Becker, G. (1965). Human Capital: A Theoretical and Empirical Analysis, with Special Reference to Education. The ANNALS of the American Academy of Political and Social Science, 360(1), 208-209. https://doi. org/10.1177/000271626536000153

Carvajal Orozco, J., \& Dávila Londoño, C. (2013). Violencia en el trabajo: investigaciones realizadas en Colombia. Sotavento M.B.A., (22), 114-124. https://revistas.uexternado. edu.co/index.php/sotavento/article/ view/3708
Colombia. Ley 776 de 2002, Diario Oficial $\S(2002)$. http://sidn.ramajudicial.gov. co/SIDN/NORMATIVA/TEXTOS COMPLETOS/7 LEYES/LEYES 2002 (731-793)/Ley 776 de 2002 (Normas sobre organización del sistema de riesgos profesionales). pdf

Colombia. Decreto 1507, Pub. L. No. Ministerio del trabajo. Decreto 1507 Por el cual se expide el, Manual Unico para la Calificación de la Pérdida de la Capacidad Laboral y Ocupacional (2014). Colombia.

Creswell, J. (2013). Research Design: Qualitative, Quantitative, and Mixed Methods Approaches. (T. Oaks, Ed.) (4th ed.). Sage publications.

Cuervo-Díaz, D., Moreno-Angarita, M., \& Elizabeth, D. (2017). Análisis de la siniestralidad en el sistema de riesgos laborales Colombiano: reflexiones desde la academia. Vnoversitas, (135), 131-163. https:// doi.org/10.11144/Javeriana.vj135. assr

Cuervo Díaz, D. E. (2016). Prevención y Manejo de la Discapacidad para Trabajar: Un Análisis al Sistema de Riesgos Laborales Colombiano. Universidad Nacional de Colombia. https://dianacuervophd.com/wpcontent/uploads/2018/09/Tesis.pdf

Espinosa, M., \& Morris, P. (2002). Calidad de vida en el trabajo: percepciones de los trabajadores. Gobierno de Chile, Dirección del Trabajo, Departamento de Estudios.

Etuknwa, A., y Daniels K, Eib C. (2019). Sustainable Return to Work: A Systematic Review Focusing on Personal and Social Factors. Journal of Occupational Rehabilitation, 29(4), 679-700. https://pubmed.ncbi.nlm. nih.gov/30767151/

Fernández, S., Tapiz, Z., Meneses, 
Galarza-Iglesias, Ana M.; Ordoñez- Hernández, Cecilia; Gómez-Salazar, Lessby

Reintegro laboral de líderes de empresas de transporte

M., Miranda, O., De Santa, M., \& López, M. (2009). Evaluación del proceso de reintegro socio-laboral en personas que han sufrido accidentes del trabajo que fueron atendidas en terapia ocupacional del Hospital del Trabajador Santiago. Ciencia \& Trabajo, 11(33), 123-129.

Guinot, J., \& Barghouti, Z. (2019). El valor de confiar en los empleados: descubriendo sus efectos sobre la participación y la motivación. UCJC Business and Society Review (Formerly Known As Universia Business Review), 16(3), 108-145. https://journals.ucjc.edu/ubr/article/ view/4007

Hernández, R., Fernandéz, C., \& Baptista, P. (2010). Metodología de la Investigación (5ta. ed.). McGrawHill Interamericana.

Husserl, E. (2000). Ideas relativas a una fenomenología pura y una filosofía fenomenológica: libro tercelo: La fenomenología y los fundamentos de las cienicas (v3). ( $\mathrm{U}$. N. de México, Ed.). México.

J Taylor R Bogdan, P. S. (2000). Introducción a los métodos cualitativos. (3ra. ed.). Paidós. https://asodea.files.wordpress. com/2009/09/taylor-s-j-bogdan-r$\underline{\text { metodologia-cualitativa.pdf }}$

León, E. (2009). El giro hermenéutico de la fenomenológica en Martín Heidegger. Polis, (22). http://journals. openedition.org/polis/2690

Leonard, V. (1989). A Heideggerian phenomenologic perspective on the concept of the person. Advances in Nursing Sciencie, 11(4), 40-55. https://doi.org/10.1097/00012272198907000-00008

Lopéz, M., \& Chicana, G. (2017). OIT. Contribuciones al debate sobre el futuro del trabajo iniciativa del centenario de la OIT la integración laboral y social de colectivos especialmente vulnerables: personas con enfermedades raras, personas con enfermedades mentales $y$ mujeres con discap. http:// scielo.isciii.es/pdf/asisna/v30n2/ colaboracion.pdf

Merlau-Ponty, M. (2000). Fenomenología de la percepción. (5ta ed.). Éditions Gallimard, Ed. Romanya.

Ministerio de la Protección Social (2019). Manual de procedimientos para la rehabilitación y reincorporación ocupacional de los trabajadores en el sistema general de riesgos profesionales. https://www. libertycolombia.com.co/sites/default/ files/2019-07/Manual\%20para $\% 20$ rehabilitacion\%202012.pdf

Ministerio de la Protección Social. Dirección General de Riesgos Profesionales. Manual de procedimientos para la rehabilitación y reincorporación ocupacional de los trabajadores en el sistema general de riesgos profesionales (2010). http://fondoriesgoslaborales. gov.co/documents/Publicaciones/ Manuales/MANUAL-PARA REHABILITACION-2012.pdf

Morse, J. (2005). Asuntos críticos en los métodos de investigación cualitativa. (U. de Antioquia, Ed.). España.

Muijzer, A., Brouwer, S., Geertzen, J. H., \& Groothoff, J. W. (2012). Exploring factors relevant in the assessment of the return-to-work process of employees on longterm sickness absence due to a depressive disorder: a focus group study. BMC Public Health, 12(103). http://www.biomedcentral.com/14712458/12/103

Ntsiea, M. V, \& van Aswegen, H. (2017). Barriers to and enablers of return to work after stroke: survivor and 
employer perceptions. Occupational Health Southern Africa, 23(2), 23-31.

Organización Mundial de la Salud, O. (2014). Documentos básicos. http:// www.who.int/es

Patton, M. (2002). Qualitative Research and Evaluation Methods. (3ra. ed.). Sage publications.

Schultz, I. Z., \& Editors, R. J. G. (2016). Handbook of Return to Work From Research to Practice Handbooks in Health, Work, and Disability. Vancouver: Springer.

Simancas, R., Sarmiento, A., Giraldo, L., \& Hernández, H. (2018). Administración del capital humano: factor de productividad empresarial en pequeñas y medianas empresas de Barranquilla. Revista Venezolana de Gerencia, 23(82), 377-391. https://www.produccioncientificaluz. org/index.php/rvg/article/ view/23754/24158

Simonelli, A. P., Camarotto, J. A., Bravo, E. S., \& Vilela, R. A. de G. (2010). Proposta de articulação entre abordagens metodo lógicas para melhoria do processo de reabilitação profissional. Rev. Bras. Saúde Ocup., 35(121), 64-73. https://doi.org/10.1590/S0303$\underline{76572010000100008}$
Trillos Ch, M. C., Tolosa G, I. A., \& Escobar F, R. Y. (2016). Modelos de rehabilitación para la intervención del desorden músculo-esquelético en trabajadores. Revista de La Universidad Industrial de Santnader. Salud, 48(4), 536-547. https://doi. org/10.18273/revsal.v48n4-2016012

Waddell, G., Dsc, C., Frcs, M. D., Aylward, M., Ffom, F., \& Frcp, F. (2010). Models of sickness and disability applied to common health problems. Abingdon: Royal Society of Medicine Press Ltd. http://www. webility.md/praxis/downloads/ Models-of-Sickness-DisabilityWaddell-and-Aylward-2010-2.pdf

Zainal,Z. (2007). Case study as a research method. Jurnal Kemanusiaan, 9(1). https://jurnalkemanusiaan.utm.my/ index.php/kemanusiaan/article/ view/165/158

Zondek, A. (2015). Manual para una Inclusión Laboral Efectiva de Personas con Discapacidad. De la deficiencia a la competencia. http:// www.sence.cl/601/articles -5284 recurso 01.pdf 